\title{
Resolvent Approach to Fourier Method in a Mixed Problem for Non-homogeneous Wave Equation
}

\begin{abstract}
V. V. Kornev ${ }^{1}$, A. P. Khromov ${ }^{2}$
${ }^{1}$ Vladimir V. Kornev, Saratov State University, 83, Astrakhanskaya str., 410012, Saratov, Russia, KornevVV @info.sgu.ru

${ }^{2}$ Avgust P. Khromov, Saratov State University, 83, Astrakhanskaya str., 410012, Saratov, Russia, KhromovAP@info.sgu.ru

Fourier method of obtaining classic solution is being justified in a mixed problem for non-homogeneous wave equation with a complex potential and fixed boundary conditions under minimal conditions on initial data. The proof is based on resolvent approach which does not need any information on eigen and associated functions of the corresponding spectral problem.
\end{abstract}

Key words: mixed problem, wave equation, Fourier method, resolvent.

The results have been obtained in the framework of the national tasks of the Ministry of Education and Science of the Russian Federation (project no. 1.1520.2014K).

\section{References}

1. Burlutskaya M. Sh., Khromov A. P. Resolvent approach in the Fourier method. Dokl. Math., 2014, vol. 90, no. 2, pp. 545-548. DOI: 10.1134/S1064562414060076.

2. Burlutskaya M. Sh., Khromov A. P. The resolvent approach for the wave equation. Comput. Math. Math. Phys., 2015, vol. 55, iss. 2, pp. 227-239. DOI: $10.1134 /$ S0965542515020050.

3. Kornev V. V., Khromov A. P. Resolvent approach to the Fourier method in a mixed problem for the wave equation. Comput. Math. Math. Phys., 2015, vol. 55, iss. 4, pp. 618-627. DOI: 10.1134/S0965542515040077.

4. Kornev V. V., Khromov A. P. A resolvent approach in the Fourier method for the wave equation: the non-selfadjoint case. Comput. Math. Math. Phys., 2015, vol. 55, iss. 7, pp. 1138-1149. DOI: 10.1134/S0965542515070088.
5. Petrovskii I. G. Lektsii ob uravneniiakh s chastnymi proizvodnymi [Lectures on Partial Differential Equations]. Moscow, Fizmatgiz, 1961. 400 p. (in Russian).

6. Chernyatin V. A. Obosnovanie metoda Fur'e v smeshannoi zadache dlya uravnenii v chastnykh proizvodnykh [Justification of the Fourier method in a mixed problem for partial differential equations]. Moscow, Moscow Univ. Press, 1991. 112 p. (in Russian).

7. Rasulov M. L. Metod konturnogo integrala [The method of the contour integral]. Moscow, Nauka, 1964. 462 p. (in Russian).

8. Vagabov A. I. Vvedenie v spektral'nuiu teoriiu differentsial'nykh operatorov [Introduction to the spectral theory of differential operators]. Rostovon-Don, Rostov Univ. Press, 1994. 106 p. (in Russian).

Please cite this article in press as:

Kornev V. V., Khromov A. P. Resolvent Approach to Fourier Method in a Mixed Problem for Non-homogeneous Wave Equation. Izv. Saratov Univ. (N. S.), Ser. Math. Mech. Inform., 2016, vol. 16, iss. 4, pp. $403-413$ (in Russian). DOI: $10.18500 / 1816-9791-2016-16-4-403-413$.

УДК 514.76

\section{TРЕХМЕРНЫЕ ОДНОРОДНЫЕ ПРОСТРАНСТВА, НЕ ДОПУСКАЮЩИЕ ИНВАРИАНТНЫХ СВЯЗНОСТЕЙ}

\section{Н. П. Можей}

Можей Наталья Павловна, кандидат фризико-математических наук, доцент кафредры программного обеспечения инфрормационных технологий, Белорусский государственный университет инфрорматики и радиоэлектроники, Минск, mozheynatalya@mail.ru

Если существует хотя бы одна инвариантная асрфинная связность на однородном пространстве, то пространство является изотропно-точным, однако обратное неверно. Возможность построения на однородном пространстве инвариантной афрфинной связности изучал П. К. Рашевский, к построениям П. К. Рашевского несколько позже пришел К. Номидзу. Цель данной работы - изучить, в каких случаях невозможно построение инвариантной асрфинной связности на трехмерном изотропно-точном однородном пространстве, и классисрицировать пространства, не допускающие инвариантных связностей. Локальная классификация однородных пространств эквивалентна описанию эффрективных пар алгебр Ли, 
соответственно найдены все изотропно-точные пары и выделены пары, на которых не существует инвариантных связностей. Особенностью представленной работы являетсяприменение чисто алгебраического подхода, сочетание различных методов дифрференциальной геометрии, теории групп Ли, алгебр Ли и однородных пространств.

Ключевые слова: инвариантная связность, однородное пространство, группа преобразований, алгебра Ли.

DOI: $10.18500 / 1816-9791-2016-16-4-413-421$

\section{ВВЕДЕНИЕ}

Понятие аффинной связности ввел Г. Вейль для построения единой теории поля. Возможностью построения на однородном пространстве инвариантной аффинной связности задавался П. К. Рашевский (см. [1] и другие его работы), к построениям П. К. Рашевского несколько позже пришел К. Номидзу (см. [2] и др.). Изучим, в каких случаях невозможно построение инвариантной аффинной связности на трехмерном изотропно-точном однородном пространстве.

Пусть $(\bar{G}, M)$ - трехмерное однородное пространство, где $\bar{G}$ - группа Ли на многообразии $M$. Зафиксируем произвольную точку $o \in M$ и обозначим через $G=\bar{G}_{o}$ стабилизатор точки $о$. Известно, что проблема классификации однородных пространств $(\bar{G}, M)$ эквивалентна классификации пар групп Ли $(\bar{G}, G)$ таких, что $G \subset \bar{G}$. Для изучения однородных пространств важно рассматривать не саму группу $\bar{G}$, а ее образ на $\operatorname{Diff}(M)$, другими словами, достаточно рассматривать только эффективные действия $\bar{G}$ на $M$. Поскольку нас интересует только проблема локальной эффективности, без ограничения общности можно считать, что $\bar{G}$ и $G$ связные. Поставим в соответствие $(\bar{G}, M)$ пару $(\overline{\mathfrak{g}}, \mathfrak{g})$ алгебр Ли, где $\overline{\mathfrak{g}}-$ алгебра Ли группы $\bar{G}$, а $\mathfrak{g}-$ подалгебра $\overline{\mathfrak{g}}$, соответствующая подгруппе $G$. Эта пара локально однозначно определяет структуру $(\bar{G}, M)$, так как два однородных пространства локально изоморфны тогда и только тогда, когда соответствующие пары алгебр Ли эквивалентны. Пара $(\overline{\mathfrak{g}}, \mathfrak{g})$ называется эффективной, если $\mathfrak{g}$ не содержит ненулевых идеалов алгебры $\overline{\mathfrak{g}}$, однородное пространство $(\bar{G}, M)$ является локально эффективным тогда и только тогда, когда соответствующая пара алгебр Ли эффективна. Изотропный $\mathfrak{g}$-модуль $\mathfrak{m}-$ это $\mathfrak{g}$-модуль $\overline{\mathfrak{g}} / \mathfrak{g}$ такой, что $x .(y+\mathfrak{g})=[x, y]+\mathfrak{g}$. Соответствующее представление $\lambda: \mathfrak{g} \rightarrow \mathfrak{g l}(\mathfrak{m})$ является изотропныц представлением пары $(\overline{\mathfrak{g}}, \mathfrak{g})$. Пара $(\overline{\mathfrak{g}}, \mathfrak{g})$ называется изотропно-точной, если ее изотропное представление - инъекция.

Разобьем решение проблемы классификации трехмерных изотропно-точных пар $(\overline{\mathfrak{g}}, \mathfrak{g})$ на этапы. Сначала классифицируем (с точностью до изоморфизма) точные трехмерные $\mathfrak{g}$-модули $U$. Это эквивалентно классификации подалгебр $\mathfrak{g l}(3, \mathbb{R})$ с точностью до сопряженности. Для каждого полученного $\mathfrak{g}$-модуля $U$ классифицируем (с точностью до эквивалентности) все такие пары $(\overline{\mathfrak{g}}, \mathfrak{g})$, что $\mathfrak{g}$-модули $\overline{\mathfrak{g}} / \mathfrak{g}$ и $U$ изоморфны. Соответствующая классификация приведена в [3]. Между инвариантными аффинными связностями на $(\bar{G}, M)$ и линейными отображениями $\Lambda: \overline{\mathfrak{g}} \rightarrow \mathfrak{g l}(\mathfrak{m})$ такими, что $\left.\Lambda\right|_{\mathfrak{g}}=\lambda$ и отображение $\Lambda$ является $\mathfrak{g}$-инвариантным, существует взаимно-однозначное соответствие (см. [2]). Будем называть такие отображения (инвариантными) аффинными связностями на паре $(\overline{\mathfrak{g}}, \mathfrak{g})$. Если возможна хотя бы одна связность на паре $(\overline{\mathfrak{g}}, \mathfrak{g})$, то такая пара является изотропно-точной (см. [4]). Аффинные связности на трехмерных однородных пространствах рассматриваются, например, в [5].

Того, что пара является изотропно-точной, не достаточно для существования инвариантных связностей. Простейший пример этого можно привести в размерности $\operatorname{codim}_{\overline{\mathfrak{g}}} \mathfrak{g}=2$. Зададим алгебру $\overline{\mathfrak{g}}$ следующей таблицей умножения:

\begin{tabular}{c|cccc} 
& $e_{1}$ & $e_{2}$ & $u_{1}$ & $u_{2}$ \\
\hline$e_{1}$ & 0 & $e_{2}$ & $2 u_{1}$ & $e_{2}+u_{2}$ \\
$e_{2}$ & $-e_{2}$ & 0 & 0 & $u_{1}$ \\
$u_{1}$ & $-2 u_{1}$ & 0 & 0 & 0 \\
$u_{2}$ & $-e_{2}-u_{2}$ & $-u_{1}$ & 0 & 0
\end{tabular},

а $\mathfrak{g}$ порождается $e_{1}$ и $e_{2}$. Прямые вычисления показывают, что не существует аффинных связностей на этой паре. Более того, полный анализ всех изотропно-точных эффективных пар коразмерности 2 (классификацию таких пар можно найти в [6]) показывает, что это единственный пример такой коразмерности. Найдем теперь все возможные пары коразмерности 3.

Будем определять пару $(\overline{\mathfrak{g}}, \mathfrak{g})$ таблицей умножения алгебры Ли $\overline{\mathfrak{g}}$. Через $\left\{e_{1}, \ldots, e_{n}\right\}$ будем обозначать базис $\overline{\mathfrak{g}}(n=\operatorname{dim} \overline{\mathfrak{g}})$. Полагаем, что алгебра Ли $\mathfrak{g}$ порождается $e_{1}, \ldots, e_{n-3}$. Пусть 
$\left\{u_{1}=e_{n-2}, u_{2}=e_{n-1}, u_{3}=e_{n}\right\}-$ базис $\mathfrak{m}$. Будем описывать аффинную связность через $\Lambda\left(e_{n-2}\right)$, $\Lambda\left(e_{n-1}\right), \Lambda\left(e_{n}\right)$ (поскольку $\left.\left.\Lambda\right|_{\mathfrak{g}}=\lambda\right)$. Для ссылки на пару будем использовать соответствующее приведенному в [3] обозначение $d . n . m$, где $d-$ размерность подалгебры, $n-$ номер подалгебры в $\mathfrak{g l}(3, \mathbb{R})$, а $m-$ номер пары $(\overline{\mathfrak{g}}, \mathfrak{g})$.

\section{1. КЛАССИФИКАЦИЯ ПАР}

Найдем изотропно-точные пары, не допускающие инвариантных связностей.

Теорема 1. Если пара $(\overline{\mathfrak{g}}, \mathfrak{g})$ не допускает инвариантных связностей, то подалгебра $\mathfrak{g} \subset \mathfrak{g l}(3, \mathbb{R})$ эквивалентна одной из следующих подалгебр:

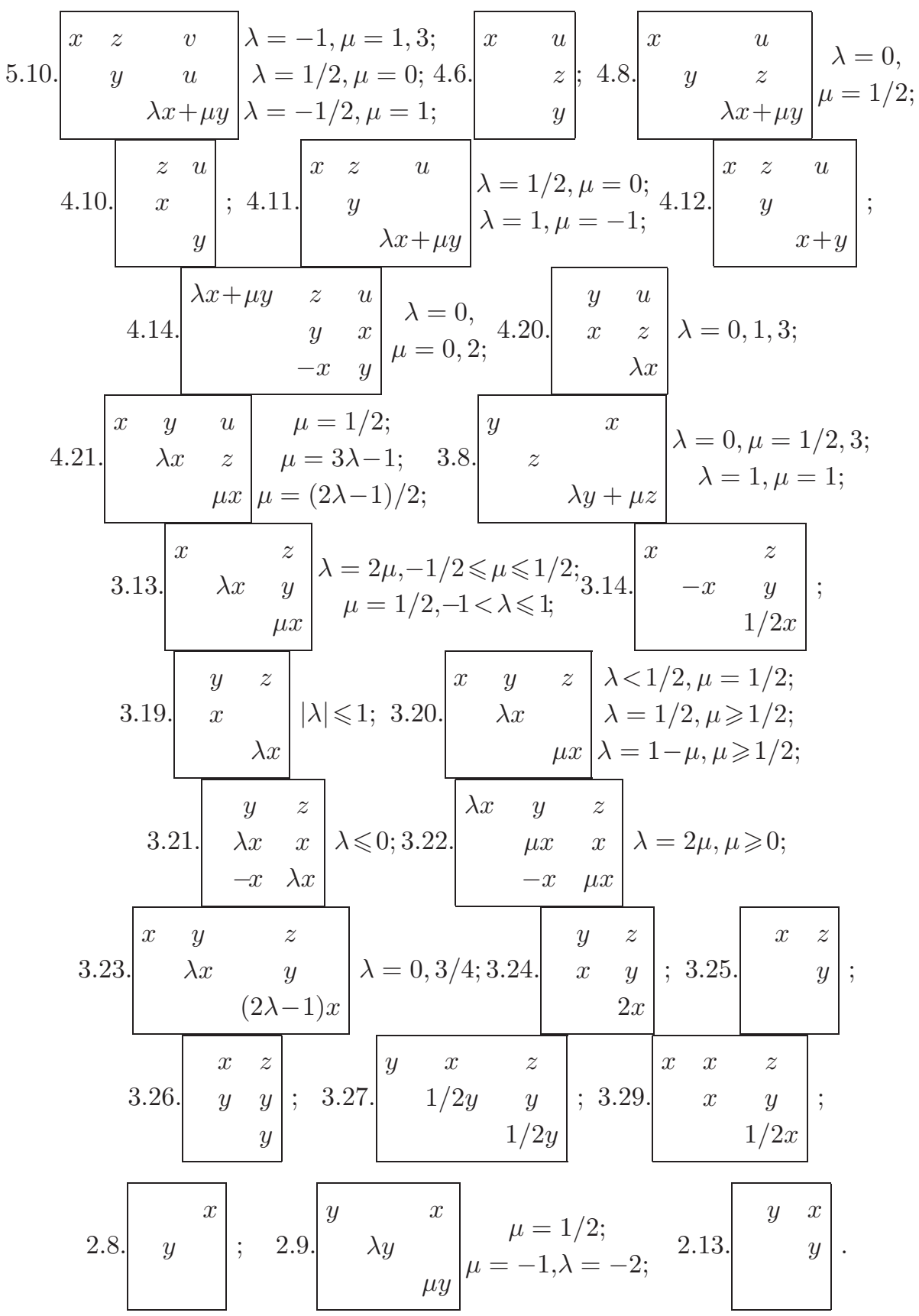

Здесь переменные обозначены латинскими буквами и принадлежат $\mathbb{R}$, параметры обозначаются маленькими греческими буквами, подалгебры с одинаковыми номерами, но различными значениями параметров, не сопряжены друг другу.

Доказательство. Для подалгебр $\mathfrak{g} \subset \mathfrak{g l}(3, \mathbb{R})$ из [3] находим изотропно-точные пары $(\overline{\mathfrak{g}}, \mathfrak{g})$ и определяем пары (и соответствующие подалгебры), не допускающие инвариантных связностей. Рас- 
смотрим, например, случай, когда изотропное представление имеет вид 3.23. Классифицируем (с точностью до эквивалентности) пары $(\overline{\mathfrak{g}}, \mathfrak{g})$ такие, что $\mathfrak{g}$-модули $\overline{\mathfrak{g}} / \mathfrak{g}$ изоморфны 3.23 .

Лемма 1. Любая пара $(\overline{\mathfrak{g}}, \mathfrak{g})$ типа 3.23 эквивалентна одной и только одной из следующих пар:

\begin{tabular}{c|cccccc}
1. & $e_{1}$ & $e_{2}$ & $e_{3}$ & $u_{1}$ & $u_{2}$ & $u_{3}$ \\
\hline$e_{1}$ & 0 & $(1-\lambda) e_{2}$ & $2(1-\lambda) e_{3}$ & $u_{1}$ & $\lambda u_{2}$ & $(2 \lambda-1) u_{3}$ \\
$e_{2}$ & $(\lambda-1) e_{2}$ & 0 & 0 & 0 & $u_{1}$ & $u_{2}$ \\
$e_{3}$ & $2(\lambda-1) e_{3}$ & 0 & 0 & 0 & 0 & $u_{1}$ \\
$u_{1}$ & $-u_{1}$ & 0 & 0 & 0 & 0 & 0 \\
$u_{2}$ & $-\lambda u_{2}$ & $-u_{1}$ & 0 & 0 & 0 & 0 \\
$u_{3}$ & $(1-2 \lambda) u_{3}$ & $-u_{2}$ & $-u_{1}$ & 0 & 0 & 0
\end{tabular},

\begin{tabular}{|c|c|c|c|c|c|c|c|c|c|c|c|c|c|c|}
\hline 2. $\lambda=3 / 5$ & $e_{1}$ & $e_{2}$ & $e_{3}$ & $u_{1}$ & $u_{2}$ & $u_{3}$ & & 3. $\lambda=1 / 2$ & $e_{1}$ & $e_{2}$ & $e_{3}$ & $u_{1}$ & $u_{2}$ & $u_{3}$ \\
\hline$e_{1}$ & 0 & $\frac{2}{5} e_{2}$ & $\frac{4}{5} e_{3}$ & $u_{1}$ & $\frac{3}{5} u_{2}$ & $\frac{1}{5} u_{3}$ & & $e_{1}$ & 0 & $\frac{1}{2} e_{2}$ & $e_{3}$ & $u_{1}$ & $\frac{1}{2} u_{2}$ & 0 \\
\hline$e_{2}$ & $-\frac{2}{5} e_{2}$ & 0 & 0 & 0 & $u_{1}$ & $u_{2}$ & & $e_{2}$ & $-\frac{1}{2} e_{2}$ & 0 & 0 & 0 & $u_{1}$ & $u_{2}$ \\
\hline$e_{3}$ & $-\frac{4}{5} e_{3}$ & 0 & 0 & 0 & 0 & $u_{1}$ & & $e_{3}$ & $-e_{3}$ & 0 & 0 & 0 & 0 & $u_{1}$ \\
\hline$u_{1}$ & $-u_{1}$ & 0 & 0 & 0 & 0 & 0 & & $u_{1}$ & $-u_{1}$ & 0 & 0 & 0 & 0 & 0 \\
\hline$u_{2}$ & $-\frac{3}{5} u_{2}$ & $-u_{1}$ & 0 & 0 & 0 & $e_{3}$ & & $u_{2}$ & $-\frac{1}{2} u_{2}$ & $-u_{1}$ & 0 & 0 & 0 & $e_{2}$ \\
\hline$u_{3}$ & $-\frac{1}{5} u_{3}$ & $-u_{2}$ & $-u_{1}$ & 0 & $-e_{3}$ & 0 & & $u_{3}$ & 0 & $-u_{2}$ & $-u_{1}$ & 0 & $-e_{2}$ & 0 \\
\hline
\end{tabular}

\begin{tabular}{c|cccccc}
$4 . \lambda=1 / 2$ & $e_{1}$ & $e_{2}$ & $e_{3}$ & $u_{1}$ & $u_{2}$ & $u_{3}$ \\
\hline$e_{1}$ & 0 & $(1 / 2) e_{2}$ & $e_{3}$ & $u_{1}$ & $(1 / 2) u_{2}$ & 0 \\
$e_{2}$ & $-(1 / 2) e_{2}$ & 0 & 0 & 0 & $u_{1}$ & $u_{2}$ \\
$e_{3}$ & $-e_{3}$ & 0 & 0 & 0 & 0 & $u_{1}$ \\
$u_{1}$ & $-u_{1}$ & 0 & 0 & 0 & 0 & 0 \\
$u_{2}$ & $-(1 / 2) u_{2}$ & $-u_{1}$ & 0 & 0 & 0 & $-e_{2}$ \\
$u_{3}$ & 0 & $-u_{2}$ & $-u_{1}$ & 0 & $e_{2}$ & 0
\end{tabular}

\begin{tabular}{c|cccccc}
$5 . \lambda=1 / 2$ & $e_{1}$ & $e_{2}$ & $e_{3}$ & $u_{1}$ & $u_{2}$ & $u_{3}$ \\
\hline$e_{1}$ & 0 & $(1 / 2) e_{2}$ & $e_{3}$ & $u_{1}$ & $(1 / 2) u_{2}$ & 0 \\
$e_{2}$ & $-(1 / 2) e_{2}$ & 0 & 0 & 0 & $u_{1}$ & $u_{2}$ \\
$e_{3}$ & $-e_{3}$ & 0 & 0 & 0 & 0 & $u_{1}$ \\
$u_{1}$ & $-u_{1}$ & 0 & 0 & 0 & 0 & $u_{1}$ \\
$u_{2}$ & $-(1 / 2) u_{2}$ & $-u_{1}$ & 0 & 0 & 0 & $\alpha e_{2}+u_{2}$ \\
$u_{3}$ & 0 & $-u_{2}$ & $-u_{1}$ & $-u_{1}$ & $-\alpha e_{2}-u_{2}$ & 0
\end{tabular},

\begin{tabular}{c|cccccc}
$6 . \lambda=3 / 4$ & $e_{1}$ & $e_{2}$ & $e_{3}$ & $u_{1}$ & $u_{2}$ & $u_{3}$ \\
\hline$e_{1}$ & 0 & $(1 / 4) e_{2}$ & $(1 / 2) e_{3}$ & $u_{1}$ & $(3 / 4) u_{2}$ & $(1 / 2) u_{3}+e_{3}$ \\
$e_{2}$ & $-(1 / 4) e_{2}$ & 0 & 0 & 0 & $u_{1}$ & $u_{2}$ \\
$e_{3}$ & $-(1 / 2) e_{3}$ & 0 & 0 & 0 & 0 & $u_{1}$ \\
$u_{1}$ & $-u_{1}$ & 0 & 0 & 0 & 0 & 0 \\
$u_{2}$ & $-(3 / 4) u_{2}$ & $-u_{1}$ & 0 & 0 & 0 & 0 \\
$u_{3}$ & $-(1 / 2) u_{3}-e_{3}$ & $-u_{2}$ & $-u_{1}$ & 0 & 0 & 0
\end{tabular}

\begin{tabular}{c|cccccc}
$7 . \lambda=0$ & $e_{1}$ & $e_{2}$ & $e_{3}$ & $u_{1}$ & $u_{2}$ & $u_{3}$ \\
\hline$e_{1}$ & 0 & $e_{2}$ & $2 e_{3}$ & $u_{1}$ & 0 & $-u_{3}$ \\
$e_{2}$ & $-e_{2}$ & 0 & 0 & $-e_{3}$ & $u_{1}-2 e_{2}$ & $u_{2}$ \\
$e_{3}$ & $-2 e_{3}$ & 0 & 0 & 0 & $-e_{3}$ & $u_{1}$ \\
$u_{1}$ & $-u_{1}$ & $e_{3}$ & 0 & 0 & $u_{1}$ & 0 \\
$u_{2}$ & 0 & $-u_{1}+2 e_{2}$ & $e_{3}$ & $-u_{1}$ & 0 & $-2 u_{3}$ \\
$u_{3}$ & $u_{3}$ & $-u_{2}$ & $-u_{1}$ & 0 & $2 u_{3}$ & 0
\end{tabular}.


Доказательство. Пусть $E=\left\{e_{1}, e_{2}, e_{3}\right\}-$ базис $\mathfrak{g}$, где

$$
e_{1}=\left(\begin{array}{ccc}
1 & 0 & 0 \\
0 & \lambda & 0 \\
0 & 0 & 2 \lambda-1
\end{array}\right), \quad e_{2}=\left(\begin{array}{ccc}
0 & 1 & 0 \\
0 & 0 & 1 \\
0 & 0 & 0
\end{array}\right), \quad e_{3}=\left(\begin{array}{ccc}
0 & 0 & 1 \\
0 & 0 & 0 \\
0 & 0 & 0
\end{array}\right)
$$

Через $\mathfrak{h}$ обозначим нильпотентную подалгебру алгебры Ли $\mathfrak{g}$, порожденную вектором $e_{1}$. Рассмотрим следующие случаи:

1. $\lambda \notin\{0,2 / 3,3 / 4\}$. Тогда

$$
\begin{gathered}
\overline{\mathfrak{g}}^{(1-\lambda)}(\mathfrak{h}) \supset \mathbb{R} e_{2}, \overline{\mathfrak{g}}^{(0)}(\mathfrak{h}) \supset \mathbb{R} e_{1}, \overline{\mathfrak{g}}^{(2-2 \lambda)}(\mathfrak{h}) \supset \mathbb{R} e_{3}, \overline{\mathfrak{g}}^{(1)}(\mathfrak{h}) \supset \mathbb{R} u_{1}, \\
\overline{\mathfrak{g}}^{(\lambda)}(\mathfrak{h}) \supset \mathbb{R} u_{2}, \overline{\mathfrak{g}}^{(2 \lambda-1)}(\mathfrak{h}) \supset \mathbb{R} u_{3}, \\
{\left[u_{1}, u_{2}\right] \in \overline{\mathfrak{g}}^{(1+\lambda)}(\mathfrak{h}),\left[u_{1}, u_{3}\right] \in \overline{\mathfrak{g}}^{(2 \lambda)}(\mathfrak{h}),\left[u_{2}, u_{3}\right] \in \overline{\mathfrak{g}}^{(3 \lambda-1)}(\mathfrak{h}) .}
\end{gathered}
$$

В силу тождества Якоби пара $(\overline{\mathfrak{g}}, \mathfrak{g})$ имеет вид

\begin{tabular}{c|cccccc} 
& $e_{1}$ & $e_{2}$ & $e_{3}$ & $u_{1}$ & $u_{2}$ & $u_{3}$ \\
\hline$e_{1}$ & 0 & $(1-\lambda) e_{2}$ & $2(1-\lambda) e_{3}$ & $u_{1}$ & $\lambda u_{2}$ & $(2 \lambda-1) u_{3}$ \\
$e_{2}$ & $(\lambda-1) e_{2}$ & 0 & 0 & 0 & $u_{1}$ & $u_{2}$ \\
$e_{3}$ & $2(\lambda-1) e_{3}$ & 0 & 0 & 0 & 0 & $u_{1}$ \\
$u_{1}$ & $-u_{1}$ & 0 & 0 & 0 & 0 & 0 \\
$u_{2}$ & $-\lambda u_{2}$ & $-u_{1}$ & 0 & 0 & 0 & $c_{3} e_{3}$ \\
$u_{3}$ & $(1-2 \lambda) u_{3}$ & $-u_{2}$ & $-u_{1}$ & 0 & $-c_{3} e_{3}$ & 0
\end{tabular},

где $c_{3}(\lambda-3 / 5)=0$, или

\begin{tabular}{c|cccccc} 
& $e_{1}$ & $e_{2}$ & $e_{3}$ & $u_{1}$ & $u_{2}$ & $u_{3}$ \\
\hline$e_{1}$ & 0 & $(1 / 2) e_{2}$ & $e_{3}$ & $u_{1}$ & $(1 / 2) u_{2}$ & 0 \\
$e_{2}$ & $-(1 / 2) e_{2}$ & 0 & 0 & 0 & $u_{1}$ & $u_{2}$ \\
$e_{3}$ & $-e_{3}$ & 0 & 0 & 0 & 0 & $u_{1}$ \\
$u_{1}$ & $-u_{1}$ & 0 & 0 & 0 & 0 & $\beta_{1} u_{1}$ \\
$u_{2}$ & $-(1 / 2) u_{2}$ & $-u_{1}$ & 0 & 0 & 0 & $c_{2} e_{2}+\beta_{1} u_{2}$ \\
$u_{3}$ & 0 & $-u_{2}$ & $-u_{1}$ & $-\beta_{1} u_{1}$ & $-c_{2} e_{2}-\beta_{1} u_{2}$ & 0
\end{tabular}.

1.1. $\lambda \neq 1 / 2$.

1.1.1. $c_{3}=0$. Тогда пара $(\overline{\mathfrak{g}}, \mathfrak{g})$ тривиальна.

1.1.2. $\lambda=3 / 5, c_{3} \neq 0$. Тогда пара $(\overline{\mathfrak{g}}, \mathfrak{g})$ эквивалентна паре $\left(\overline{\mathfrak{g}}_{2}, \mathfrak{g}_{2}\right)$, эквивалентность показывает отображение $\pi: \overline{\mathfrak{g}}_{2} \rightarrow \overline{\mathfrak{g}}$, где $\pi\left(e_{1}\right)=e_{1}, \pi\left(e_{2}\right)=c_{3}{ }^{-1 / 3} e_{2}, \pi\left(e_{3}\right)=c_{3}{ }^{-2 / 3} e_{3}, \pi\left(u_{1}\right)=c_{3}{ }^{-4 / 3} u_{1}$, $\pi\left(u_{2}\right)=\left(1 / c_{3}\right) u_{2}, \pi\left(u_{3}\right)=c_{3}{ }^{-2 / 3} u_{3}$.

1.2. $\lambda=1 / 2$.

1.2.1. $\beta_{1}=c_{2}=0$. Тогда пара $(\overline{\mathfrak{g}}, \mathfrak{g})$ тривиальна.

1.2.2. $\beta_{1}=0, c_{2} \neq 0$. Тогда пара $(\overline{\mathfrak{g}}, \mathfrak{g})$ эквивалентна паре $\left(\overline{\mathfrak{g}}_{i}, \mathfrak{g}_{i}\right)$, где $i=3$ или $i=4$ (отображение $\pi: \overline{\mathfrak{g}}_{i} \rightarrow \overline{\mathfrak{g}}$, где $\pi\left(e_{j}\right)=e_{j}, j=\overline{1,3}, \pi\left(u_{k}\right)=\left|c_{2}\right|^{-1 / 2} u_{k}, k=\overline{1,3}$, если $c_{2}>0$, то $i=3$, если $c_{2}<0$, то $i=4)$

1.2.3. $\beta_{1} \neq 0$. Тогда пара $(\overline{\mathfrak{g}}, \mathfrak{g})$ эквивалентна паре $\left(\overline{\mathfrak{g}}_{5}, \mathfrak{g}_{5}\right)$ посредством отображения $\pi: \overline{\mathfrak{g}}_{5} \rightarrow \overline{\mathfrak{g}}$, $\pi\left(e_{i}\right)=e_{i}, i=\overline{1,3}, \pi\left(u_{j}\right)=\left(1 / \beta_{1}\right) u_{j}, j=\overline{1,3}$.

2. $\lambda=3 / 4$. Имеем $\overline{\mathfrak{g}}^{(1 / 4)}(\mathfrak{h})=\mathbb{R} e_{2}, \overline{\mathfrak{g}}^{(0)}(\mathfrak{h})=\mathbb{R} e_{1}, \overline{\mathfrak{g}}^{(1)}(\mathfrak{h})=\mathbb{R} u_{1}, \overline{\mathfrak{g}}^{(3 / 4)}(\mathfrak{h})=\mathbb{R} u_{2}$,

$$
\overline{\mathfrak{g}}^{(1 / 2)}(\mathfrak{h})=\mathbb{R} u_{3} \oplus \mathbb{R} e_{3},\left[u_{1}, u_{2}\right] \in \overline{\mathfrak{g}}^{(7 / 4)}(\mathfrak{h}) \Rightarrow\left[u_{1}, u_{2}\right]=0,\left[u_{1}, u_{3}\right] \in \overline{\mathfrak{g}}^{(3 / 2)}(\mathfrak{h}) \Rightarrow\left[u_{1}, u_{3}\right]=0,
$$

$\left[u_{2}, u_{3}\right] \in \overline{\mathfrak{g}}^{(5 / 4)}(\mathfrak{h}) \Rightarrow\left[u_{2}, u_{3}\right]=0$. 
В силу тождества Якоби пара $(\overline{\mathfrak{g}}, \mathfrak{g})$ имеет вид

\begin{tabular}{c|cccccc} 
& $e_{1}$ & $e_{2}$ & $e_{3}$ & $u_{1}$ & $u_{2}$ & $u_{3}$ \\
\hline$e_{1}$ & 0 & $(1 / 4) e_{2}$ & $(1 / 2) e_{3}$ & $u_{1}$ & $(3 / 4) u_{2}$ & $(1 / 2) u_{3}+p e_{3}$ \\
$e_{2}$ & $-(1 / 4) e_{2}$ & 0 & 0 & 0 & $u_{1}$ & $u_{2}$ \\
$e_{3}$ & $-(1 / 2) e_{3}$ & 0 & 0 & 0 & 0 & $u_{1}$ \\
$u_{1}$ & $-u_{1}$ & 0 & 0 & 0 & 0 & 0 \\
$u_{2}$ & $-(3 / 4) u_{2}$ & $-u_{1}$ & 0 & 0 & 0 & 0 \\
$u_{3}$ & $-(1 / 2) u_{3}-p e_{3}$ & $-u_{2}$ & $-u_{1}$ & 0 & 0 & 0
\end{tabular}.

2.1. $p=0$. Тогда пара $(\overline{\mathfrak{g}}, \mathfrak{g})$ тривиальна.

2.2. $p \neq 0$. Тогда пара $(\overline{\mathfrak{g}}, \mathfrak{g})$ эквивалентна паре $\left(\overline{\mathfrak{g}}_{6}, \mathfrak{g}_{6}\right)$, эквивалентность показывает отображение $\pi: \overline{\mathfrak{g}}_{6} \rightarrow \overline{\mathfrak{g}}, \pi\left(e_{i}\right)=e_{i}, i=\overline{1,3}, \pi\left(u_{j}\right)=(1 / p) u_{j}, j=\overline{1,3}$.

3. $\lambda=0$. Тогда $\overline{\mathfrak{g}}^{(1)}(\mathfrak{h})=\mathbb{R} e_{2} \oplus \mathbb{R} u_{1}, \overline{\mathfrak{g}}^{(0)}(\mathfrak{h})=\mathbb{R} e_{1} \oplus \mathbb{R} u_{2}, \overline{\mathfrak{g}}^{(2)}(\mathfrak{h})=\mathbb{R} e_{3}, \overline{\mathfrak{g}}^{(-1)}(\mathfrak{h})=\mathbb{R} u_{3}$ и $\left[u_{1}, u_{2}\right] \in \overline{\mathfrak{g}}^{(1)}(\mathfrak{h}) \Rightarrow\left[u_{1}, u_{2}\right]=a_{2} e_{2}+\alpha_{1} u_{1}, \quad\left[u_{1}, u_{3}\right] \in \overline{\mathfrak{g}}^{(0)}(\mathfrak{h}) \Rightarrow\left[u_{1}, u_{3}\right]=b_{1} e_{1}+\beta_{2} u_{2}$, $\left[u_{2}, u_{3}\right] \in \overline{\mathfrak{g}}^{(-1)}(\mathfrak{h}) \Rightarrow\left[u_{2}, u_{3}\right]=\gamma_{3} u_{3}$. Учитывая тождество Якоби, получаем, что пара $(\overline{\mathfrak{g}}, \mathfrak{g})$ имеет вид

\begin{tabular}{c|cccccc} 
& $e_{1}$ & $e_{2}$ & $e_{3}$ & $u_{1}$ & $u_{2}$ & $u_{3}$ \\
\hline$e_{1}$ & 0 & $e_{2}$ & $2 e_{3}$ & $u_{1}$ & 0 & $-u_{3}$ \\
$e_{2}$ & $-e_{2}$ & 0 & 0 & $p e_{3}$ & $u_{1}+2 p e_{2}$ & $u_{2}$ \\
$e_{3}$ & $-2 e_{3}$ & 0 & 0 & 0 & $p e_{3}$ & $u_{1}$ \\
$u_{1}$ & $-u_{1}$ & $-p e_{3}$ & 0 & 0 & $-p u_{1}$ & 0 \\
$u_{2}$ & 0 & $-u_{1}-2 p e_{2}$ & $-p e_{3}$ & $p u_{1}$ & 0 & $2 p u_{3}$ \\
$u_{3}$ & $u_{3}$ & $-u_{2}$ & $-u_{1}$ & 0 & $-2 p u_{3}$ & 0
\end{tabular}.

3.1. $p=0$. Тогда пара $(\overline{\mathfrak{g}}, \mathfrak{g})$ тривиальна.

3.2. $p \neq 0$. Тогда пара $(\overline{\mathfrak{g}}, \mathfrak{g})$ эквивалентна паре $\left(\overline{\mathfrak{g}}_{7}, \mathfrak{g}_{7}\right)$ посредством отображения $\pi: \overline{\mathfrak{g}}_{7} \rightarrow \overline{\mathfrak{g}}$ $\pi\left(e_{1}\right)=e_{1}, \pi\left(u_{1}\right)=u_{1}, \pi\left(e_{2}\right)=p e_{2}, \pi\left(u_{2}\right)=(1 / p) u_{2}, \pi\left(e_{3}\right)=p^{2} e_{3}, \pi\left(u_{3}\right)=\left(1 / p^{2}\right) u_{3}$.

4. $\lambda=2 / 3$. Имеем $\overline{\mathfrak{g}}^{(1 / 3)}(\mathfrak{h})=\mathbb{R} e_{2} \oplus \mathbb{R} u_{3}, \overline{\mathfrak{g}}^{(0)}(\mathfrak{h})=\mathbb{R} e_{1}, \overline{\mathfrak{g}}^{(2 / 3)}(\mathfrak{h})=\mathbb{R} e_{3} \oplus \mathbb{R} u_{2}, \overline{\mathfrak{g}}^{(1)}(\mathfrak{h})=\mathbb{R} u_{1}$, $\left[u_{1}, u_{2}\right] \in \overline{\mathfrak{g}}^{(5 / 3)}(\mathfrak{h}) \Rightarrow\left[u_{1}, u_{2}\right]=0,\left[u_{1}, u_{3}\right] \in \overline{\mathfrak{g}}^{(4 / 3)}(\mathfrak{h}) \Rightarrow\left[u_{1}, u_{3}\right]=0,\left[u_{2}, u_{3}\right] \in \overline{\mathfrak{g}}^{(1)}(\mathfrak{h}) \Rightarrow\left[u_{2}, u_{3}\right]=\gamma_{1} u_{1}$. В силу тождества Якоби пара $(\overline{\mathfrak{g}}, \mathfrak{g})$ принимает вид

\begin{tabular}{c|cccccc} 
& $e_{1}$ & $e_{2}$ & $e_{3}$ & $u_{1}$ & $u_{2}$ & $u_{3}$ \\
\hline$e_{1}$ & 0 & $(1 / 3) e_{2}$ & $(2 / 3) e_{3}$ & $u_{1}$ & $(2 / 3) u_{2}$ & $(1 / 3) u_{3}$ \\
$e_{2}$ & $-(1 / 3) e_{2}$ & 0 & 0 & 0 & $u_{1}$ & $u_{2}$ \\
$e_{3}$ & $-(2 / 3) e_{3}$ & 0 & 0 & 0 & 0 & $u_{1}$ \\
$u_{1}$ & $-u_{1}$ & 0 & 0 & 0 & 0 & 0 \\
$u_{2}$ & $-(2 / 3) u_{2}$ & $-u_{1}$ & 0 & 0 & 0 & $\gamma_{1} u_{1}$ \\
$u_{3}$ & $-(1 / 3) u_{3}$ & $-u_{2}$ & $-u_{1}$ & 0 & $-\gamma_{1} u_{1}$ & 0
\end{tabular}

Тогда пара $(\overline{\mathfrak{g}}, \mathfrak{g})$ эквивалентна тривиальной паре $\left(\overline{\mathfrak{g}}_{1}, \mathfrak{g}_{1}\right)$, эквивалентность показывает отображение $\pi: \overline{\mathfrak{g}}_{1} \rightarrow \overline{\mathfrak{g}}, \pi\left(e_{i}\right)=e_{i}, i=\overline{1,3}, \pi\left(u_{1}\right)=u_{1}, \pi\left(u_{2}\right)=u_{2}, \pi\left(u_{3}\right)=u_{3}+\gamma_{1} e_{2}$.

Теперь осталось показать, что полученные пары не эквивалентны друг другу.

Поскольку $\operatorname{dim} D^{2} \overline{\mathfrak{g}}_{1} \neq \operatorname{dim} D^{2} \overline{\mathfrak{g}}_{2}$, видим, что пары $\left(\overline{\mathfrak{g}}_{1}, \mathfrak{g}_{1}\right)$ и $\left(\overline{\mathfrak{g}}_{2}, \mathfrak{g}_{2}\right)$ не эквивалентны.

Пусть $\lambda=3 / 4$. Рассмотрим гомоморфизмы $f_{i}: \overline{\mathfrak{g}}_{i} \rightarrow \mathfrak{g l}(5, \mathbb{R}), i=1,6$, где $f_{i}(x)$ - матрица отображения $\left.a d\right|_{D \overline{\mathfrak{g}}_{i}} x$ в базисе $\left\{e_{2}, e_{3}, u_{1}, u_{2}, u_{3}\right\}$ пространства $D \overline{\mathfrak{g}}_{i}$. Так как подалгебры $f_{i}\left(\overline{\mathfrak{g}}_{i}\right)$ не сопряжены, можно заключить, что пары $\left(\overline{\mathfrak{g}}_{1}, \mathfrak{g}_{1}\right)$ и $\left(\overline{\mathfrak{g}}_{6}, \mathfrak{g}_{6}\right)$ не эквивалентны. Поскольку $\operatorname{dim} D^{2} \overline{\mathfrak{g}}_{1} \neq \operatorname{dim} D^{2} \overline{\mathfrak{g}}_{7}$, видим, что пары $\left(\overline{\mathfrak{g}}_{1}, \mathfrak{g}_{1}\right)$ и $\left(\overline{\mathfrak{g}}_{7}, \mathfrak{g}_{7}\right)$ не эквивалентны.

Пусть $\lambda=1 / 2$. Рассмотрим гомоморфизмы $f_{i}: \overline{\mathfrak{g}}_{i} \rightarrow \mathfrak{g l}(4, \mathbb{R}), i=1, \overline{3,5}$, где $f_{i}(x)$ - матрица отображения $\left.a d\right|_{D \overline{\mathfrak{g}}_{i}} x$ в базисе $\left\{e_{2}, e_{3}, u_{1}, u_{2}\right\}$ пространства $\overline{\mathfrak{g}}_{i}$. Поскольку подалгебры $f_{i}\left(\overline{\mathfrak{g}}_{i}\right)$ не сопряжены, то пары $\left(\overline{\mathfrak{g}}_{1}, \mathfrak{g}_{1}\right),\left(\overline{\mathfrak{g}}_{3}, \mathfrak{g}_{3}\right),\left(\overline{\mathfrak{g}}_{4}, \mathfrak{g}_{4}\right),\left(\overline{\mathfrak{g}}_{5}, \mathfrak{g}_{5}\right)$ не эквивалентны друг другу.

Лемма 2. Если пара $(\overline{\mathfrak{g}}, \mathfrak{g})$ не допускает инвариантных аффинных связностей, а $\mathfrak{g}$ имеет вид 3.23, то $(\overline{\mathfrak{g}}, \mathfrak{g})$ эквивалентна одной из пар 3.23.6, 3.23.7. 
Доказательство. Пусть $(\overline{\mathfrak{g}}, \mathfrak{g})$ - трехмерное однородное пространство типа $3.23 .3,\left.\Lambda\right|_{\mathfrak{g}}-$ изотропное представление $\mathfrak{g}$. Пусть здесь и далее

$$
\Lambda\left(u_{1}\right)=\left(\begin{array}{lll}
p_{1,1} & p_{1,2} & p_{1,3} \\
p_{2,1} & p_{2,2} & p_{2,3} \\
p_{3,1} & p_{3,2} & p_{3,3}
\end{array}\right), \quad \Lambda\left(u_{2}\right)=\left(\begin{array}{ccc}
q_{1,1} & q_{1,2} & q_{1,3} \\
q_{2,1} & q_{2,2} & q_{2,3} \\
q_{3,1} & q_{3,2} & q_{3,3}
\end{array}\right), \quad \Lambda\left(u_{3}\right)=\left(\begin{array}{lll}
r_{1,1} & r_{1,2} & r_{1,3} \\
r_{2,1} & r_{2,2} & r_{2,3} \\
r_{3,1} & r_{3,2} & r_{3,3}
\end{array}\right) .
$$

Отображение $\Lambda-\mathfrak{g}$-инвариантно. Следовательно, из $\left[\Lambda\left(e_{3}\right), \Lambda\left(u_{1}\right)\right]=\Lambda\left(\left[e_{3}, u_{1}\right]\right)$ получаем $\left[\Lambda\left(e_{3}\right), \Lambda\left(u_{1}\right)\right]=0$. Тогда имеем $p_{3,1}=p_{3,2}=p_{2,1}=0, p_{3,3}=p_{1,1}$. $\left[\Lambda\left(e_{2}\right), \Lambda\left(u_{1}\right)\right]=\Lambda\left(\left[e_{2}, u_{1}\right]\right)$, откуда получаем $\left[\Lambda\left(e_{2}\right), \Lambda\left(u_{1}\right)\right]=0, p_{2,3}=p_{1,2}, p_{2,2}=p_{1,1}$. $\left[\Lambda\left(e_{1}\right), \Lambda\left(u_{1}\right)\right]=\Lambda\left(u_{1}\right)$, тогда $p_{1,1}=p_{1,2}=0$. Если $\left[\Lambda\left(e_{3}\right), \Lambda\left(u_{2}\right)\right]=0$, то $q_{3,1}=q_{3,2}=q_{2,1}=0, q_{3,3}=q_{1,1} \cdot\left[\Lambda\left(e_{2}\right), \Lambda\left(u_{2}\right)\right]=\Lambda\left(u_{1}\right)$, получаем $q_{2,3}=q_{1,2}+p_{1,3}, q_{2,2}=q_{1,1} .\left[\Lambda\left(e_{1}\right), \Lambda\left(u_{2}\right)\right]=(1 / 2) \Lambda\left(u_{2}\right)$, откуда $q_{1,1}=q_{1,3}=0$. Если $\left[\Lambda\left(e_{3}\right), \Lambda\left(u_{3}\right)\right]=\Lambda\left(u_{1}\right)$, то $r_{3,1}=r_{3,2}=r_{2,1}=0, r_{3,3}=r_{1,1}+p_{1,3}$. $\left[\Lambda\left(e_{2}\right), \Lambda\left(u_{3}\right)\right]=\Lambda\left(u_{2}\right)$, следовательно, $r_{2,2}=r_{1,1}, q_{1,2}=0, r_{2,3}=r_{1,2}$. $\left[\Lambda\left(e_{1}\right), \Lambda\left(u_{3}\right)\right]=0$, имеем $r_{1,2}=r_{1,3}=0$, аффинная связность существует и имеет вид

$$
\Lambda\left(u_{1}\right)=\left(\begin{array}{ccc}
0 & 0 & p_{1,3} \\
0 & 0 & 0 \\
0 & 0 & 0
\end{array}\right), \quad \Lambda\left(u_{2}\right)=\left(\begin{array}{ccc}
0 & 0 & 0 \\
0 & 0 & p_{1,3} \\
0 & 0 & 0
\end{array}\right), \quad \Lambda\left(u_{3}\right)=\left(\begin{array}{ccc}
r_{1,1} & 0 & 0 \\
0 & r_{1,1} & 0 \\
0 & 0 & r_{1,1}+p_{1,3}
\end{array}\right) .
$$

Тензор кривизны имеет вид

$$
\begin{gathered}
R\left(u_{1}, u_{2}\right)=0, \quad R\left(u_{1}, u_{3}\right)=\left[\Lambda\left(u_{1}\right), \Lambda\left(u_{3}\right)\right]-\Lambda\left(\left[u_{1}, u_{3}\right]\right)=\left(\begin{array}{ccc}
0 & 0 & p_{1,3}{ }^{2} \\
0 & 0 & 0 \\
0 & 0 & 0
\end{array}\right), \\
R\left(u_{2}, u_{3}\right)=\left(\begin{array}{ccc}
0 & -1 & 0 \\
0 & 0 & p_{1,3}{ }^{2}-1 \\
0 & 0 & 0
\end{array}\right),
\end{gathered}
$$

тензор кручения

$$
\begin{gathered}
T\left(u_{1}, u_{2}\right)=0, \quad T\left(u_{1}, u_{3}\right)=\Lambda\left(u_{1}\right)\left(u_{3}\right)_{\mathfrak{m}}-\Lambda\left(u_{3}\right)\left(u_{1}\right)_{\mathfrak{m}}-\left[u_{1}, u_{3}\right]_{\mathfrak{m}}=\left(p_{1,3}-r_{1,1}, 0,0\right), \\
T\left(u_{2}, u_{3}\right)=\Lambda\left(u_{2}\right)\left(u_{3}\right)_{\mathfrak{m}}-\Lambda\left(u_{3}\right)\left(u_{2}\right)_{\mathfrak{m}}-\left[u_{2}, u_{3}\right]_{\mathfrak{m}}=\left(0, p_{1,3}-r_{1,1}, 0\right) .
\end{gathered}
$$

Пусть $(\overline{\mathfrak{g}}, \mathfrak{g})$ - трехмерное однородное пространство типа 3.23.6, отображение $\Lambda \mathfrak{g}$-инвариантно, следовательно, $\left[\Lambda\left(e_{3}\right), \Lambda\left(u_{1}\right)\right]=\Lambda\left(\left[e_{3}, u_{1}\right]\right)$. Откуда $\left[\Lambda\left(e_{3}\right), \Lambda\left(u_{1}\right)\right]=0$. Имеем $p_{3,1}=p_{3,2}=p_{2,1}=0$, $p_{3,3}=p_{1,1}$. Из $\left[\Lambda\left(e_{2}\right), \Lambda\left(u_{1}\right)\right]=\Lambda\left(\left[e_{2}, u_{1}\right]\right)$ получаем $\left[\Lambda\left(e_{2}\right), \Lambda\left(u_{1}\right)\right]=0, p_{2,3}=p_{1,2}, p_{2,2}=p_{1,1}$. $\left[\Lambda\left(e_{1}\right), \Lambda\left(u_{1}\right)\right]=\Lambda\left(u_{1}\right)$, тогда $p_{1,1}=0$. Если $\left[\Lambda\left(e_{3}\right), \Lambda\left(u_{2}\right)\right]=0$, то $q_{3,1}=q_{3,2}=q_{2,1}=0$, $q_{3,3}=q_{1,1} \cdot\left[\Lambda\left(e_{2}\right), \Lambda\left(u_{2}\right)\right]=\Lambda\left(u_{1}\right)$, следовательно, $p_{1,2}=0, q_{2,3}=q_{1,2}+p_{1,3}, q_{2,2}=q_{1,1}$. Если $\left[\Lambda\left(e_{3}\right), \Lambda\left(u_{3}\right)\right]=\Lambda\left(u_{1}\right)$, то $r_{3,1}=r_{3,2}=r_{2,1}=0, r_{3,3}=r_{1,1}+p_{1,3} .\left[\Lambda\left(e_{2}\right), \Lambda\left(u_{3}\right)\right]=\Lambda\left(u_{2}\right)$, тогда $r_{2,2}=r_{1,1}, q_{1,2}=q_{1,1}=0, r_{2,3}=r_{1,2}+q_{1,3}$. Если $\left[\Lambda\left(e_{1}\right), \Lambda\left(u_{3}\right)\right]=0$, то $r_{1,2}=r_{1,3}=0$. Учитывая $\left[\Lambda\left(e_{1}\right), \Lambda\left(u_{3}\right)\right]=(1 / 2) \Lambda\left(u_{3}\right)+\Lambda\left(e_{3}\right)$, имеем

$$
\left(\begin{array}{ccc}
-1 / 2 r_{1,1} & -1 / 4 r_{1,2} & -1 \\
0 & -1 / 2 r_{1,1} & -1 / 4 r_{1,2}-1 / 4 q_{1,3} \\
0 & 0 & -1 / 2 r_{1,1}-1 / 2 p_{1,3}
\end{array}\right)=0
$$

т. е. у уравнения нет решений, пара $(\overline{\mathfrak{g}}, \mathfrak{g})$ не допускает аффинных связностей. Прямыми вычислениями получаем результаты для остальных пар типа 3.23 .

Аналогично, если пара $(\overline{\mathfrak{g}}, \mathfrak{g})$ не допускает аффинных связностей, а $\mathfrak{g}$ имеет тип 3.19 , то $(\overline{\mathfrak{g}}, \mathfrak{g})$ эквивалентна одной из пар 3.19.2, 3.19.3, 3.19.5, 3.19.6, 3.19.8, 3.19.9, 3.19.10, 3.19.11, 3.19.12, 3.19.13, 3.19 .15 [3]. Например, если $(\overline{\mathfrak{g}}, \mathfrak{g})$ - трехмерное однородное пространство типа 3.19 .2 , то

$$
\Lambda\left(e_{1}\right)=\left(\begin{array}{ccc}
0 & 0 & 0 \\
0 & 1 & 0 \\
0 & 0 & \lambda
\end{array}\right), \quad \Lambda\left(e_{2}\right)=\left(\begin{array}{ccc}
0 & 1 & 0 \\
0 & 0 & 0 \\
0 & 0 & 0
\end{array}\right), \quad \Lambda\left(e_{3}\right)=\left(\begin{array}{ccc}
0 & 0 & 1 \\
0 & 0 & 0 \\
0 & 0 & 0
\end{array}\right)
$$


$\left(\left.\Lambda\right|_{\mathfrak{g}}=\lambda\right)$. Отображение $\Lambda-\mathfrak{g}$-инвариантно. Если $\left[\Lambda\left(e_{3}\right), \Lambda\left(u_{1}\right)\right]=\Lambda\left(\left[e_{3}, u_{1}\right]\right)$, то

$$
\left[\Lambda\left(e_{3}\right), \Lambda\left(u_{1}\right)\right]=0, \quad\left(\begin{array}{ccc}
p_{3,1} & p_{3,2} & p_{3,3}-p_{1,1} \\
0 & 0 & -p_{2,1} \\
0 & 0 & -p_{3,1}
\end{array}\right)=0, \quad p_{3,1}=p_{3,2}=p_{2,1}=0, \quad p_{3,3}=p_{1,1}
$$

Если $\left[\Lambda\left(e_{2}\right), \Lambda\left(u_{1}\right)\right]=\Lambda\left(\left[e_{2}, u_{1}\right]\right)$, то

$$
\left[\Lambda\left(e_{2}\right), \Lambda\left(u_{1}\right)\right]=-\Lambda\left(e_{2}\right), \quad\left(\begin{array}{ccc}
0 & p_{2,2}-p_{1,1}-1 & p_{2,3} \\
0 & 0 & 0 \\
0 & 0 & 0
\end{array}\right)=0, \quad p_{2,2}=p_{1,1}+1, \quad p_{2,3}=0
$$

Если $\left[\Lambda\left(e_{1}\right), \Lambda\left(u_{1}\right)\right]=\Lambda\left(\left[e_{1}, u_{1}\right]\right)$, то $\left[\Lambda\left(e_{1}\right), \Lambda\left(u_{1}\right)\right]=0, p_{1,2}=0$.

Поскольку $\left[\Lambda\left(e_{3}\right), \Lambda\left(u_{2}\right)\right]=0, q_{3,1}=q_{3,2}=q_{2,1}=0, q_{3,3}=q_{1,1}$; если $\left[\Lambda\left(e_{2}\right), \Lambda\left(u_{2}\right)\right]=\Lambda\left(u_{1}\right)$, то $\left(\begin{array}{ccc}-p_{1,1} & q_{2,2}-q_{1,1} & q_{2,3}-p_{1,3} \\ 0 & -p_{1,1}-1 & 0 \\ 0 & 0 & -p_{1,1}\end{array}\right)=0$, уравнение не имеет решений, пара $(\overline{\mathfrak{g}}, \mathfrak{g})$ не допускает аффинных связностей.

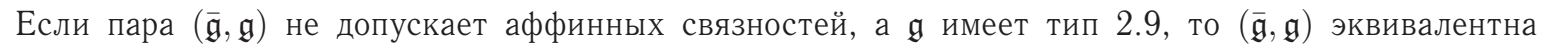
одной из пар $2.9 .8,2.9 .9,2.9 .10,2.9 .17$. Например, если $(\overline{\mathfrak{g}}, \mathfrak{g})-$ трехмерное однородное пространство типа 2.9.17 [3], то

$$
\Lambda\left(e_{1}\right)=\left(\begin{array}{ccc}
1 & 0 & 0 \\
0 & -2 & 0 \\
0 & 0 & -1
\end{array}\right), \quad \Lambda\left(e_{2}\right)=\left(\begin{array}{lll}
0 & 0 & 1 \\
0 & 0 & 0 \\
0 & 0 & 0
\end{array}\right)
$$

(так как $\left.\left.\Lambda\right|_{\mathfrak{g}}=\lambda\right)$. Если $\Lambda-\mathfrak{g}$-инвариантно, то $\left[\Lambda\left(e_{2}\right), \Lambda\left(u_{1}\right)\right]=\Lambda\left(\left[e_{2}, u_{1}\right]\right)$. Следовательно, $\left[\Lambda\left(e_{2}\right), \Lambda\left(u_{1}\right)\right]=0, p_{3,1}=p_{3,2}=p_{2,1}=0, p_{3,3}=p_{1,1}$. Из $\left[\Lambda\left(e_{1}\right), \Lambda\left(u_{1}\right)\right]=\Lambda\left(\left[e_{1}, u_{1}\right]\right)$ следует $\left[\Lambda\left(e_{1}\right), \Lambda\left(u_{1}\right)\right]=\Lambda\left(u_{1}\right)$, тогда $p_{1,1}=p_{1,2}=p_{1,3}=p_{2,2}=p_{2,3}=0$. Из $\left[\Lambda\left(e_{2}\right), \Lambda\left(u_{2}\right)\right]=\Lambda\left(e_{1}\right)$ следует, что $\left(\begin{array}{ccc}q_{3,1}-1 & q_{3,2} & q_{3,3}-q_{1,1} \\ 0 & 2 & -q_{2,1} \\ 0 & 0 & -q_{3,1}+1\end{array}\right)=0$, пара $(\overline{\mathfrak{g}}, \mathfrak{g})$ не допускает аффинных связностей. Прямыми вычислениями получаем результаты в остальных случаях.

Аналогично, если пара $(\overline{\mathfrak{g}}, \mathfrak{g})$ не допускает аффинных связностей, а $\mathfrak{g}$ имеет тип 4.6 , то $(\overline{\mathfrak{g}}, \mathfrak{g})$ эквивалентна паре 4.6.2 [3]. Если пара $(\overline{\mathfrak{g}}, \mathfrak{g})$ не допускает аффинных связностей, а $\mathfrak{g}$ имеет тип 5.10, то $(\overline{\mathfrak{g}}, \mathfrak{g})$ эквивалентна одной из пар $5.10 .3,5.10 .4,5.10 .7,5.10 .8,5.10 .9$. Если $\mathfrak{g}$ имеет тип 4.8 , то $(\overline{\mathfrak{g}}, \mathfrak{g})$ эквивалентна одной из пар 4.8.7, 4.8.8. Если $\mathfrak{g}$ имеет тип 4.10, то $(\overline{\mathfrak{g}}, \mathfrak{g})$ эквивалентна 4.10.2, 4.10.3. Если $\mathfrak{g}$ имеет тип 4.11 , то $(\overline{\mathfrak{g}}, \mathfrak{g})$ эквивалентна $4.11 .6,4.11 .7,4.11 .8,4.11 .9$. Если $\mathfrak{g}$ имеет тип 4.12 , то $(\overline{\mathfrak{g}}, \mathfrak{g})$ эквивалентна 4.12.2. Если $\mathfrak{g}$ имеет тип 4.14, то $(\overline{\mathfrak{g}}, \mathfrak{g})$ эквивалентна 4.14.2, 4.14.3, 4.14.4, 4.14.5. Если $\mathfrak{g}$ имеет тип 4.20, то $(\overline{\mathfrak{g}}, \mathfrak{g})$ эквивалентна 4.20.6, 4.20.7, 4.20.8, 4.20.9, 4.20.10, 4.20.11, 4.20.12, 4.20.13. Если $\mathfrak{g}$ имеет тип 4.21, то $(\overline{\mathfrak{g}}, \mathfrak{g})$ эквивалентна 4.21.8, 4.21.9, 4.21.10, 4.21.12, 4.21.13, 4.21.14, $4.21 .15,4.21 .16,4.21 .17,4.21 .18,4.21 .19,4.21 .20,4.21 .21,4.21 .22,4.21 .23$. Если g имеет тип 3.8, то $(\overline{\mathfrak{g}}, \mathfrak{g})$ эквивалентна $3.8 .4,3.8 .5,3.8 .6$. Если $\mathfrak{g}$ имеет тип 3.13 , то $(\overline{\mathfrak{g}}, \mathfrak{g})$ эквивалентна $3.13 .7,3.13 .14$, $3.13 .15,3.13 .16,3.13 .17,3.13 .18,3.13 .19,3.13 .20,3.13 .21,3.13 .22,3.13 .23,3.13 .24,3.13 .25$. Если $\mathfrak{g}$ имеет тип 3.14 , то $(\overline{\mathfrak{g}}, \mathfrak{g})$ эквивалентна 3.14 .4 . Если $\mathfrak{g}$ имеет тип 3.20, то $(\overline{\mathfrak{g}}, \mathfrak{g})$ эквивалентна 3.20.6, 3.20.7, $3.20 .8,3.20 .9,3.20 .10,3.20 .16,3.20 .17,3.20 .18,3.20 .19,3.20 .28,3.20 .29$. Если g имеет тип 3.21 , то $(\overline{\mathfrak{g}}, \mathfrak{g})$ эквивалентна $3.21 .2,3.21 .3,3.21 .4,3.21 .5$. Если $\mathfrak{g}$ имеет тип 3.22 , то $(\overline{\mathfrak{g}}, \mathfrak{g})$ эквивалентна 3.22 .2 . Если $\mathfrak{g}$ имеет тип 3.24 , то $(\overline{\mathfrak{g}}, \mathfrak{g})$ эквивалентна 3.24 .2 . Если $\mathfrak{g}$ имеет тип 3.25 , то $(\overline{\mathfrak{g}}, \mathfrak{g})$ эквивалентна 3.25 .9 , 3.25.10, 3.25.11, 3.25.12, 3.25.13, 3.25.14, 3.25.15, 3.25.16, 3.25.17, 3.25.18, 3.25.19, 3.25.20,

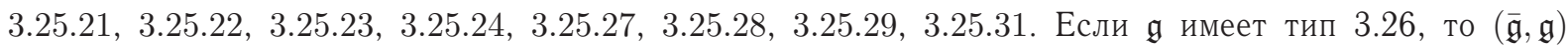
эквивалентна 3.26.2. Если $\mathfrak{g}$ имеет тип 3.27, то $(\overline{\mathfrak{g}}, \mathfrak{g})$ эквивалентна 3.27.5. Если $\mathfrak{g}$ имеет тип 3.29, то $(\overline{\mathfrak{g}}, \mathfrak{g})$ эквивалентна 3.29.2. Если $\mathfrak{g}$ имеет тип 2.8 , то $(\overline{\mathfrak{g}}, \mathfrak{g})$ эквивалентна $2.8 .8,2.8 .9,2.8 .10,2.8 .11$. Если $\mathfrak{g}$ имеет тип 2.13 , то $(\overline{\mathfrak{g}}, \mathfrak{g})$ эквивалентна 2.13 .9 .

Проводя аналогичные рассуждения для всех подалгебр, получаем, что других трехмерных изотропно-точных однородных пространств, не допускающих инвариантных связностей, кроме представленных выше, не существует. 


\section{Библиограсрический список}

1. Рашевский П. К. Риманова геометрия и тензорный анализ. М. : Наука, 1967. 664 с.

2. Nomizu K. Invariant affine connections on homogeneous spaces // Amer. J. Math. 1954. Vol. 76, № 1. P. 33-65. DOI: 10.2307/2372398.

3. Можей Н. П. Трехмерные изотропно-точные однородные пространства и связности на них. Казань : Изд-во Казан. ун-та, 2015. 394 с.

4. Kobayashi S., Nomizu K. Foundations of differential geometry. N. Y. : John Wiley and Sons, 1963. Vol. 1. 340 p.; 1969. Vol. 2. 485 p.
5. Mozhey N. Invariant affine connections on threedimensional homogeneous spaces with nonsolvable transformation group // Lobachevskii J. Math. 2014. Vol. 35, № 3. P. 218-240. DOI: 10.1134/S1995080214030093.

6. Komrakov B., Tchourioumov A., Doubrov B. Two-dimensional homogeneous spaces. Preprint series: Pure mathematics. 1993. № 17. 142 p. URL: http://urn.nb.no/URN:NBN:no-47681 (дата обращения: 15.11.2015).

Образец для цитирования:

Можей Н. П. Трехмерные однородные пространства, не допускающие инвариантных связностей // Изв. Сарат. ун-та. Нов. сер. Сер. Математика. Механика. Информатика. 2016. Т. 16, вып. 4. C. 413-421. DOI: 10.18500/18169791-2016-16-4-413-421.

\title{
Three-dimensional Homogeneous Spaces, Not Admitting Invariant Connections
}

\begin{abstract}
N. P. Mozhey
Natalya P. Mozhey, Belarussian State University of Informatics and Radioelectronics, 6, P. Brovki str., 220013, Minsk, Belarus, mozheynatalya@mail.ru

The purpose of the work is the classification of three-dimensional isotropy-faithful homogeneous spaces, not admitting invariant connections. The local classification of homogeneous spaces is equivalent to the description of effective pairs of Lie algebras. If there exists at least one invariant connection then the space is isotropy-faithful, but the isotropy-faithfulness is not sufficient for the space in order to have invariant connections. The peculiarity of techniques presented in the work is the application of purely algebraic approach, the compound of different methods of differential geometry, theory of Lie groups, Lie algebras and homogeneous spaces.

Key words: invariant connection, homogeneous space, transformation group, Lie algebra.
\end{abstract}

\section{References}

1. Rashevskii P. K. Rimanova geometriia i tenzornyi analiz [Riemannian geometry and tensor analysis]. Moscow, Nauka, 1967, 664 p. (in Russian).

2. Nomizu K. Invariant affine connections on homogeneous spaces. Amer. J. Math., 1954, vol. 76, no. 1, pp. 33-65. DOI: 10.2307/2372398.

3. Mozhey N. P. Trekhmernye izotropno-tochnye odnorodnye prostranstva $i$ sviaznosti na nikh [Three-dimensional isotropy-faithful homogeneous spaces and connections on them]. Kazan', Kazan' Univ. Press, 2015, 394 p. (in Russian).

4. Kobayashi S., Nomizu K. Foundations of differen- tial geometry. New York, John Wiley and Sons, 1963, vol. 1, 340 p.; 1969, vol. 2, 485 p.

5. Mozhey N. Invariant affine connections on three-dimensional homogeneous spaces with nonsolvable transformation group. Lobacherskii J. Math., 2014, vol. 35, no. 3, pp. 218-240. DOI: 10.1134/S1995080214030093.

6. Komrakov B., Tchourioumov A., Doubrov B. Twodimensional homogeneous spaces. Preprint series: Pure mathematics, 1993, no. 17. 142 p. Available at: http://urn.nb.no/URN:NBN:no-4768 (accessed 15.11.2015).

Please cite this article in press as:

Mozhey N. P. Three-dimensional Homogeneous Spaces, Not Admitting Invariant Connections. Izv. Saratov Univ. (N.S.), Ser. Math. Mech. Inform., 2016, vol. 16, iss. 4, pp. 413-421 (in Russian). DOI: 10.18500/1816-9791-2016-164-413-421. 